\title{
The Positive Lyapunov Exponent Of The Matrix With The Exponential Rotation
}

\author{
Kai Tao \\ College of Sciences, Hohai University, 1 Xikang Road Nanjing Jiangsu 210098 P.R.China \\ ktao@hhu.edu.cn
}

Keywords: Positive Lyapunov exponent, Exponential rotation, Harmonic measure

Abstract. This paper studies the Lyapunov exponent defined by the matrix with the exponential rotation. The author applies the theory of subharmonic functions to prove that if the coupling number is big enough, then the Lyapunov exponent is positive.

\section{Introduction}

In this paper, we consider the Lyapunov exponent defined by the following matrix:

$$
A(x, E):=\left(\begin{array}{cccc}
\lambda v_{11}(x)-E & v_{12}(x) & \cdots & v_{1 m}(x) \\
v_{21}(x) & v_{22}(x) & \cdots & v_{2 m}(x) \\
\vdots & \vdots & \ddots & \vdots \\
v_{m 1}(x) & v_{m 2}(x) & \cdots & v_{m m}(x)
\end{array}\right)
$$

where every $v_{i j}(x), i, j=1,2 \cdots, m$, is an analytic function on $T:=\mathbb{R} \backslash \mathbb{Z}$. Then

$$
M_{n}(x, E)=\prod_{j=n-1}^{0} A\left(T^{j}(x), E\right)=\prod_{j=n-1}^{0}\left(\begin{array}{cccc}
\lambda v_{11}\left(T^{j}(x)\right)-E & v_{12}\left(T^{j}(x)\right) & \cdots & v_{1 m}\left(T^{j}(x)\right) \\
v_{21}\left(T^{j}(x)\right) & v_{22}\left(T^{j}(x)\right) & \cdots & v_{2 m}\left(T^{j}(x)\right) \\
\vdots & \vdots & \ddots & \vdots \\
v_{m 1}\left(T^{j}(x)\right) & v_{m 2}\left(T^{j}(x)\right) & \cdots & v_{m m}\left(T^{j}(x)\right)
\end{array}\right)
$$

is called the transfer matrix of (1) and here $\mathrm{T}$ is the exponential rotation:

$$
T^{j}(x)=x+\omega^{j},
$$

where ${ }^{\omega}$ is a irrational number. Now we can define the Lyapunov exponent:

$$
L(E)=\liminf _{n \rightarrow \infty} \frac{1}{n} \int_{\mathrm{T}} \log \left\|M_{n}(x, E)\right\| d x,
$$

where $\left\|M_{n}(x, E)\right\|=\max _{\|v\|_{2}=1}\left\|M_{n}(x, E) v\right\|_{2}$. In this paper, we will show that this Lyapunov exponent is positive if the coupling number is big enough. For the detail, please see the main theorem at the end of this paper.

Actually, this question comes from some famous discrete operators, such like the Schrodinger operator, the Jacobi operator, the extended Harper's model and so on, which have many applications in Physics. Also, the property of matrix multiplication is a significant topic in computer theory. So, similar questions were studied in [1], [2] and [5].

\section{Positive Lyapunov Exponent}

In this section, we show the proof of the main theorem. First, we recommend the following lemmas from [2] and [4], which will be applied in the proof of the positive Lyapunov exponent:

Lemma 2: For all $0<\delta<\rho$, there is an $\epsilon$ such that

$$
\inf _{E_{1}} \sup _{\substack{i \leq y \leq i \\ x \in[0,1]}}\left|v(x+i y)-E_{1}\right|>\epsilon .
$$

Lemma 3: Let $u: \Omega \rightarrow[-\infty,+\infty)$ be an upper semi-continuous function. Then $\mathrm{u}(\mathrm{z})$ is a subharmonic function on $\Omega$, if and only if for any Jordan subdomain $\Omega^{\prime}$ satisfying $\overline{\Omega^{\prime}} \subset \Omega$ ' and any $z \in \Omega^{\prime}$, it has

$$
u(z) \leq \int_{\partial \Omega^{\prime}} u(\zeta) d \mu_{\zeta}\left(z, \partial \Omega^{\prime}, \Omega^{\prime}\right),
$$


where $\mu\left(z, \partial \Omega^{\prime}, \Omega^{\prime}\right)$ is the harmonic measure of $\partial \Omega^{\prime}$ at $z \in \Omega^{\prime}$.

Now without loss of generality, let $\lambda>0$. We all know that if $\mathrm{v}$ is real analytic function on $\mathrm{T}$, then there exists some ${ }^{\rho_{v}>0}$ : such that

$$
v(x)=\sum_{k \in Z} \hat{v}(k) e^{2 \pi i k x}, \text { with }|\hat{v}(k)| \lesssim e^{-p_{u}|k|} .
$$

So, it has a holomorphic extension

$$
v(z)=\sum_{k \in Z} \hat{v}(k) e^{2 \pi i k z}
$$

to the strip $|\operatorname{Im} z|<\frac{\rho_{v}}{10}$, satisfying

$$
|v(z)| \leq \sum_{k \in \mathbb{Z}}|\hat{v}(k)| e^{2 \pi|k||m z|}<\sum_{k \in \mathbb{Z}} e^{-\rho_{\mid}|k|} e^{\rho^{\beta}|k| \frac{\pi}{10}}<C_{\gamma} .
$$

Thus, for any $1 \leq i, j \leq m$, i it has

$$
v_{i j}(x)=\sum_{k \in \mathbb{Z}} \hat{v}_{i j}(k) e^{2 \pi i k x} \text {, with }\left|\hat{v}_{i j}(k)\right| \lesssim e^{-p_{i j}|k|},
$$

Then define

$$
C_{i j}=\sup _{|\operatorname{lm} z| \leq \frac{\rho_{y}}{10}}\left|v_{i j}(z)\right|, 1 \leq i, j \leq m, C=\max _{i, j} C_{i j} \text { and } \rho=\min _{i, j} \rho_{i j} .
$$

Then, by uniformly hyperbolic, we can assume that $|E|<m C \lambda$ in the following paper. Thus, $M_{n}(z, E)$ defined in (2), is analytic on ${ }^{|\operatorname{Im} z|<\frac{\rho}{10}}$ with fixed $\omega:$ and E, and $\left\|M_{n}(z, E)\right\| \leq(2 m C \lambda)^{n}$. So, define

$$
u_{n}(z):=\frac{1}{n} \log \left\|M_{n}(z, E)\right\|,
$$

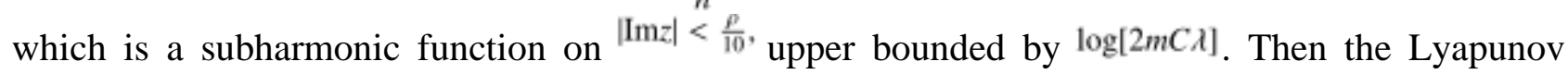
exponent becomes

$$
L(E)=\liminf _{n} L_{n}(E),
$$

where

$$
L_{n}(E)=\int_{\mathbb{T}} u_{n}(x) d x
$$

Fix $0<\delta \ll \rho$ : and $\epsilon$ satisfying Lemma2. Define

$$
\lambda_{0}=100 m C \epsilon^{-100}>0
$$

and let $\lambda>\lambda_{0}>0$. Then, for fixed $\mathrm{E}$, there is $\frac{\delta}{2}<y_{0}<\delta$ such that

$$
\inf _{x \in[0,1]}\left|v_{11}\left(x+i y_{0}\right)-\frac{E}{\lambda}\right|>\epsilon \text {. }
$$

Thus, by periodic,

$$
\inf _{x \in \mathbb{R}}\left|\lambda v_{11}\left(x+i y_{0}\right)-E\right|>\lambda \epsilon>100 m C \epsilon^{-99}>100 m C .
$$

Define

$$
M_{n-1}\left(i y_{0}, E\right)\left(\begin{array}{c}
1 \\
0 \\
\vdots \\
0
\end{array}\right)=\left(\begin{array}{c}
w_{1}^{n-1} \\
w_{2}^{n-1} \\
\vdots \\
w_{m}^{n-1}
\end{array}\right),
$$

Then 
(9)

$$
\begin{aligned}
\left(\begin{array}{c}
w_{1}^{n} \\
w_{2}^{n} \\
\vdots \\
w_{m}^{n}
\end{array}\right)= & \left(\begin{array}{cccc}
\lambda v_{11}\left(i y_{0}+\omega^{n}\right)-E & v_{12}\left(i y_{0}+\omega^{n}\right) & \cdots & v_{1 m}\left(i y_{0}+\omega^{n}\right) \\
v_{21}\left(i y_{0}+\omega^{n}\right) & v_{22}\left(i y_{0}+\omega^{n}\right) & \cdots & v_{2 m}\left(i y_{0}+\omega^{n}\right) \\
\vdots & \vdots & \ddots & \vdots \\
v_{m 1}\left(i y_{0}+\omega^{n}\right) & v_{m 2}\left(i y_{0}+\omega^{n}\right) & \cdots & v_{m m}\left(i y_{0}+\omega^{n}\right)
\end{array}\right)\left(\begin{array}{c}
w_{1}^{n-1} \\
w_{2}^{n-1} \\
\vdots \\
w_{m}^{n-1}
\end{array}\right) \\
= & \left(\begin{array}{c}
\left(\lambda v_{11}\left[i y_{0}+\omega^{n}\right]-E\right) w_{1}^{n-1}+\sum_{j=2}^{m} v_{1 j}\left[i y_{0}+\omega^{n}\right] w_{j}^{n-1} \\
\sum_{j=1}^{m} v_{2 j}\left[i y_{0}+\omega^{n}\right] w_{j}^{n-1} \\
\vdots \\
\sum_{j=1}^{m} v_{m j}\left[i y_{0}+\omega^{n}\right] w_{j}^{n-1}
\end{array}\right) .
\end{aligned}
$$

Now we use induction to show that

$$
\left|w_{1}^{n}\right| \geq\left|w_{j}^{n}\right|, j=2, \cdots, m \text {, and }\left|w_{1}^{n}\right| \geq(\lambda \epsilon-m C)\left|w_{1}^{n-1}\right| \geq(\lambda \epsilon-m C)^{n}, n \geq 1 .
$$

Because $w_{1}^{0}=1, w_{j}^{0}=0, j=2, \cdots, m$, so it yields

$$
\left|w_{1}^{1}\right|=\lambda \epsilon>100 m C,\left|w_{j}^{1}\right|<C, j=2, \cdots, m,
$$

which satisfy (10) for $n=1$. Then let $n=t$ with

$$
\left|w_{1}^{t}\right| \geq\left|w_{j}^{t}\right|, j=1, \cdots, m \text {, and }\left|w_{1}^{t}\right|>(\lambda \epsilon-m C)\left|w_{1}^{t-1}\right|>(\lambda \epsilon-m C)^{t} .
$$

Due to (9) and (11), it has

$$
\begin{aligned}
& \left|w_{1}^{t+1}\right| \geq(\lambda \epsilon-m C) w_{1}^{t}>(\lambda \epsilon-m C)^{t+1}, \\
& \left|w_{j}^{t+1}\right| \leq m C\left|w_{1}^{t}\right|<99 m C\left|w_{1}^{t}\right| \leq(\lambda \epsilon-m C)\left|w_{1}^{t}\right| \leq\left|w_{1}^{t+1}\right|, j=2, \cdots, m,
\end{aligned}
$$

which also satisfy (10) for $\mathrm{n}=\mathrm{t}+1$. Thus, the expression (10) holds for any $n \geq 1$. Then

$$
\left\|M_{n}\left(i y_{0}, E\right)\right\|>(\lambda \epsilon-m C)^{n} \text { and } u_{n}\left(i y_{0}\right)>\log (\lambda \epsilon-m C) \text {. }
$$

Write $H_{s}$ for the strip $\left\{z=x+i y: 0<y<\frac{\rho}{10}\right\}$ and denote $\mu_{s}\left(i y_{0}, E_{s}, H_{s}\right)$ the harmonic measure of $E_{s}$ at $i y_{0} \in \mathbb{H}_{s}$, where $E_{s} \subset \partial \mathbb{H}_{s}=\mathbb{R} \cup\left[y=\frac{\rho}{10}\right]$. Thus, by [3],

$$
\mu_{s}\left[y=\frac{\rho}{10}\right]=\frac{10 \pi y_{0}}{\pi \rho}<\frac{10 \delta}{\rho} \text { and }\left.\frac{d \mu_{s}(x)}{d x}\right|_{y=0}<\frac{y_{0}}{x^{2}+y_{0}^{2}} .
$$

So, by subharmonic and Lemma 3, it yields

$$
\begin{aligned}
\log (\lambda \epsilon-m C)<u_{n}\left(i y_{0}\right) & \leq \int_{[y=0] \cup\left[y=\frac{\rho}{10}\right]} u_{n}(z) \mu_{s}(d z) \\
& =\int_{y=0} u_{n}(x) \mu_{s}(d x)+\int_{y=\frac{\rho}{10}} u_{n}(x+i y) \mu_{s}(d x) \\
& \leq \int_{\mathbb{R}} u_{n}(x) \frac{y_{0}}{x^{2}+y_{0}^{2}} d x+\frac{10 \delta}{\rho}\left[\sup _{y=\frac{\rho}{10}} u_{n}(x+i y)\right] \\
& \leq \int_{\mathbb{R}} u_{n}(x) \frac{y_{0}}{x^{2}+y_{0}^{2}} d x+\frac{\bar{C} \delta}{\rho} \log \lambda .
\end{aligned}
$$

Then

$$
\begin{aligned}
L_{n}(E)=\int_{0}^{1} u_{n}(\theta) d \theta & \geq \frac{y_{0}}{2} \int_{0}^{1} u_{n}(\theta)\left(\sum_{k \in \mathbb{Z}} \frac{y_{0}}{y_{0}^{2}+(\theta+k)^{2}}\right) d \theta \\
& \geq \frac{y_{0}}{2}\left(\log (\lambda \epsilon-m C)-\frac{10 \delta}{\rho} \log (2 m C \lambda)\right) \\
& \geq \frac{\delta}{4}\left(\frac{1}{2} \log \lambda-\frac{1}{100} \log \lambda_{0}\right) \\
& \geq c \log \lambda, \forall n \geq 1 .
\end{aligned}
$$

where the inequality (12) comes from the setting of $\lambda_{0}$ and $\delta \ll \rho$. Note that the small constant c depends on all $v_{i j}$. Thus, it has

$$
L(E)=\liminf _{n} L_{n}(E) \geq c \log \lambda,
$$


which proves the following main theorem:

Main Theorem: There exists $\lambda_{0}>0$ such that for any irrational ${ }^{\omega}$, if the coupling number $|\lambda|>\lambda_{0}$, then

$$
L(E) \geq c \log |\lambda| \text { for all } E,
$$

where ${ }^{\lambda_{0}}$ and c depend only on $v_{i j}, i, j=1,2, \cdots, m$.

\section{References}

[1]. J.Bourgain, Positive Lyapunov exponents for most energy. Geometric aspects of functional analysis, 37-66, Lecture Note in Math.17410,Springer, Berlin, 2000.

[2]. J.Bourgain and M.Goldstein, On nonperturbative localization with quasi-periodic potential. Ann. of Math. (2) 1102 (2000), no.3, 8310--879.

[3]. J.Garnett and D.Marshall, Harmonic Measure. Cambridge University Press, 2005.

[4]. T.Ransford, Potential Theory in the Complex Plane, London Mathematical Society Student Texts 28, Cambridge University Press,1995.

[5]. E. Sorets and T. Spencer, Positive Lyapunov exponent for Schrodinger operators with quasi-periodic potential. Comm. Math. Phys.142, (1991),543-566. 